\title{
Clinical Investigations
}

\section{Bronchoalveolar Lavage Cellular Patterns in Monoclonal Antibody-Induced Lung Disease}

\author{
Shikha Gupta ${ }^{a}$ Patricia Smith ${ }^{a} \quad$ Homer L. Twigg III ${ }^{a}$ Chadi A. Hage ${ }^{b}$ \\ a Pulmonary-Critical Care Medicine, and b Thoracic Transplantation Program, Pulmonary-Critical Care Medicine, \\ Indiana University School of Medicine, Indianapolis, Ind., USA
}

\section{Key Words}

Monoclonal antibody · Interstitial lung disease .

Bronchoalveolar lavage

\begin{abstract}
Background: The increase in the use of monoclonal antibodies ( $m A B$ ) as a targeted therapy for a variety of diseases has been accompanied by an increase in reports of interstitial lung abnormalities in treated patients. Objective: Bronchoalveolar lavage (BAL) is routinely performed in these patients to rule out infection, so we sought to determine the BAL cellular pattern in individuals with $\mathrm{mAB}$-induced lung disease (mAB-ILD). Methods: We utilized a case-control study design. Among patients treated with $m A B$, cases were defined as those with otherwise-unexplained interstitial lung abnormalities, which resolved after cessation of treatment, while controls were defined as those with interstitial abnormalities clearly explained by other etiologies. Results: From 2000 to 2012, we identified 9 cases and 7 controls. The mean age of the cases was $62.6 \pm 26$ years and 6 were female. The most common radiographic finding was diffuse ground-glass opacities. The most common BAL cellular pattern was mixed inflammation with moderate lymphocytic and mild neutrophilic alveolitis. The cases had a higher
\end{abstract}

mean lymphocyte count than the controls (40.1 \pm 32.6 vs. $13.1 \pm 25.5, p=0.008)$. The rest of the BAL cellular analyses were similar between the 2 groups. The median CD4:CD8 ratio in 7 patients with $>15 \%$ lymphocytes was 0.9 (0.6-3). There was no significant difference in the CD4:CD8 ratio between the 2 groups. Conclusions: Mixed inflammation with moderate lymphocytic and mild neutrophilic alveolitis is the most common BAL cellular pattern in patients with mAB-ILD. Such findings may be useful for the early identification of $\mathrm{mAB}-$ ILD.

(c) 2014 S. Karger AG, Basel

\section{Introduction}

Monoclonal antibodies (mAB) directed against various components of the immune system have gained in importance in the treatment of autoimmune disorders and neoplasms in recent years [1]. With the increasing use of $\mathrm{mAB}$, there have been more case reports and case series describing adverse effects which range from as-

These data were presented as a poster at the ATS meeting in May 2013 in Philadelphia, Pa., USA.

\section{KARGER}

E-Mail karger@karger.com

www.karger.com/res
C 2014 S. Karger AG, Basel

0025-7931/14/0883-0185\$39.50/0
Chadi A. Hage, MD

Indiana University Health, Thoracic Transplantation Program

Methodist Professional Center-1, 1801 North Senate Boulevard, Suite 230

Indianapolis, IN 46202 (USA)

E-Mail chage@iu.edu 
Table 1. Patient characteristics in the $\mathrm{mAB}-\mathrm{ILD}$ group

\begin{tabular}{|c|c|c|c|c|c|c|c|}
\hline No. & Age/sex & Race & Smoker & $\mathrm{mAB}$ agent & Underlying disease & Presenting complaints & CT findings \\
\hline 1 & $82 / \mathrm{M}$ & Caucasian & No & cetuximab & metastatic colon cancer & dyspnea, fever & $\begin{array}{l}\text { patchy GGOs, predominantly } \\
\text { in upper lobes }\end{array}$ \\
\hline 2 & $53 / \mathrm{F}$ & Caucasian & Quit & infliximab & inflammatory bowel disease & dyspnea, fever, cough & diffuse GGOs, worse at bases \\
\hline 3 & $56 / \mathrm{F}$ & Caucasian & No & rituximab & non-Hodgkin lymphoma & dyspnea, fever, cough & diffuse GGOs \\
\hline 4 & $68 / \mathrm{F}$ & Caucasian & No & rituximab & non-Hodgkin lymphoma & dyspnea & minimal bilateral GGOs \\
\hline 5 & $66 / \mathrm{F}$ & Caucasian & No & bevacizumab & metastatic breast cancer & dyspnea & diffuse GGOs and consolidation \\
\hline 6 & $35 / \mathrm{F}$ & Caucasian & No & trastuzumab & invasive breast cancer & dyspnea & diffuse GGOs \\
\hline 7 & $67 / \mathrm{M}$ & Caucasian & No & rituximab & chronic lymphocytic leukemia & cough & diffuse GGOs \\
\hline 8 & $66 / \mathrm{M}$ & Hispanic & No & rituximab & non-Hodgkin lymphoma & dyspnea, hemoptysis & diffuse GGOs \\
\hline 9 & $71 / \mathrm{M}$ & Afro-American & Quit & rituximab & Factor VIII deficiency & dyspnea & diffuse GGOs \\
\hline
\end{tabular}

ymptomatic increases in autoantibody titers [2] to lifethreatening systemic complications.

The first case of $\mathrm{mAB}$-induced lung disease (mABILD) was reported in 2003 with rituximab [3]. Since then, over 150 cases have been reported in the literature. In 2006, a multicenter project called BIOGEAS was created to collect data on the use of $\mathrm{mAB}$ in adult patients with systemic autoimmune diseases, and to study their adverse effects. Data on 118 patients with mAB-ILD-related side effects were reported. The most common $\mathrm{mAB}$ agents implicated were rituximab and infliximab [1].

Bronchoalveolar lavage (BAL) is widely used in the evaluation of interstitial lung disease (ILD), and recent guidelines from the American Thoracic Society highlighted the utility of BAL cellular patterns in the diagnostic evaluation of this disease [4]. BAL has been used in the evaluation of patients with suspected drug-induced lung disease, as it helps to support clinical-pathological patterns and exclude other causes such as infections or neoplasms [5]. However, among patients with $\mathrm{mAB}$-ILD, the description of BAL abnormalities has been limited to case reports and small case series, with no systematic evaluation having been performed.

In this study, we describe the BAL cellular profiles in patients with $\mathrm{mAB}-\mathrm{ILD}$, and compare them to patients with lung disease due to other etiologies. We also describe the demographic characteristics of patients with mAB-ILD.

\section{Materials and Methods}

\section{Study Design and Data Collection}

A case-control study was conducted on all patients receiving $\mathrm{mAB}$ therapy for autoimmune or neoplastic diseases who developed pulmonary symptoms associated with chest radiograph abnormalities and underwent bronchoscopy with BAL. To be included in the study, a portion of the BAL had to be sent to the clinical bronchoalveolar lavage laboratory at the Indiana University. The rest of the BAL was sent to the microbiology laboratory at Indiana University Health for bacterial, fungal and viral studies. We included patients from the Methodist, Wishard and Indiana University Hospitals from January 2000 to December 2012. Patients who were not on $\mathrm{mAB}$ or did not have complete records were excluded. BAL was processed as previously described [6]. Cell counts and differentials were determined. CD4 and CD8 T cell subsets were quantified using flow cytometry in BAL samples with $>15 \%$ lymphocytes [6]. The study was approved by the Indiana University Institutional Review Board.

\section{Definition of $m A B-I L D$}

For patients presenting with any radiographic pattern of ILD, the definition of mAB-ILD for the purpose of our study was according to the diagnosis of $\mathrm{mAB}$-ILD made by the treating pulmonologist. The diagnosis was made after review of clinical data and lung imaging, and after excluding causes such as infections, neoplasms, hemorrhage and adult respiratory distress syndrome. $\mathrm{Pa}$ tients on $\mathrm{mAB}$ with radiographic abnormalities clearly explained by other etiologies were used as controls.

\section{Statistical Analysis}

Nonparametric analysis was used. Results were expressed as median and interquartile range (IQR). The continuous variables were analyzed by means of the $\chi^{2}$ test. For noncontinuous variables, the Mann-Whitney rank sum test was conducted. Statistical significance was defined as a $\mathrm{p}$ value $<0.05$. Analysis was performed using SPSS software, version 9.

\section{Results}

A total of 38 patient records were reviewed. Sixteen patients were excluded because they were not on $\mathrm{mAB}$ and another 6 due to the unavailability of complete medical records. A total of 9 cases and 7 controls were identified. Of the 7 controls, 4 had pneumonia and 1 patient each had lymphangitic spread of an underlying breast cancer, graft-versus-host disease and sarcoidosis. 


\section{Baseline Characteristics}

In patients with $\mathrm{mAB}-\mathrm{ILD}$, the mean age was 62.6 $( \pm 13)$ years. Six $(66.7 \%)$ were female and $7(77.8 \%)$ were Caucasian. None of the patients were active smokers. The most common $\mathrm{mAB}$ used was rituximab in 5(55.5\%) patients. Cetuximab, infliximab, bevacizumab and trastuzumab were used in 1 patient each. The most common indication for use was malignancy $(\mathrm{n}=7 ; 77.8 \%)$. The baseline characteristics of these patients are shown in table 1 . With regard to age, sex, race, smoking status and the $\mathrm{mAB}$ used, patients were similar to controls (table 2).

\section{Clinical Presentation and Management}

The most common presenting symptom in the cases was dyspnea in $8(88.9 \%)$ patients. Cough and fever were present in 3 (33.3\%) patients. The most common radiographic finding was diffuse ground-glass opacities (GGOs; table 1). Chest computed tomography and bronchoscopy with cellular and microbiologic analysis of the BAL fluid were obtained for all patients in both groups. Lung biopsies were obtained for 3 patients with mAB-ILD (transbronchial biopsy in 2 and open-lung biopsy in 1) and 4 controls (transbronchial biopsies in 2, transbronchial and open-lung biopsy in 1 and openlung biopsy and autopsy in 1). In the mAB-ILD group, $\mathrm{mAB}$ was discontinued in all patients. All patients received corticosteroids, though variable doses and regimens were used. With the above management, all patients in the $\mathrm{mAB}$ group experienced a clinical and radiographic improvement. One of the patients was rechallenged and redeveloped similar symptoms. Discontinuation of $\mathrm{mAB}$ and corticosteroids resulted in complete recovery.

\section{BAL Cellular Analysis}

The most common BAL cellular pattern in patients with $\mathrm{mAB}$-ILD was mixed inflammation with moderate lymphocytic and mild neutrophilic alveolitis. The median cell counts in the $\mathrm{mAB}$ group were $38 \%$ macrophages (IQR 30-46), 42\% lymphocytes (IQR 40-52), $17 \%$ neutrophils (IQR 14-22) and 1\% eosinophils (IQR $0-4)$. The median CD4:CD8 ratio in 7 patients with $>15 \%$ lymphocytes was 0.9 (IQR $0.6-3$ ). The median lymphocyte count in BAL was higher in the patients with $\mathrm{mAB}-\mathrm{ILD}$ than in controls ( 42 vs. $10 \%, \mathrm{p}=0.007$; fig. 1). The rest of the BAL cellular analysis in both the groups was not significantly different. There was no significant difference in the CD4:CD8 ratio between the groups (table 3).

Monoclonal Antibody-Induced Lung Disease
Table 2. Patient characteristics

\begin{tabular}{lccl}
\hline & $\begin{array}{l}\text { mAB-ILD } \\
(\mathrm{n}=9)\end{array}$ & $\begin{array}{l}\text { Other } \\
\text { etiologies } \\
(\mathrm{n}=7)\end{array}$ & p value \\
\hline Median age, years (IQR) & $66(56-68)$ & $55(47-60)$ & 0.06 \\
Females, n & $6(67 \%)$ & $4(57 \%)$ & 0.7 \\
Caucasians, $\mathrm{n}$ & $7(78 \%)$ & $6(86 \%)$ & 0.7 \\
Nonsmokers, $\mathrm{n}$ & $7(78 \%)$ & $6(86 \%)$ & 0.7 \\
On rituximab, $\mathrm{n}$ & $5(56 \%)$ & $2(29 \%)$ & 0.3 \\
On other mAB, n & $4(44 \%)$ & $5(71 \%)$ & \\
\hline
\end{tabular}

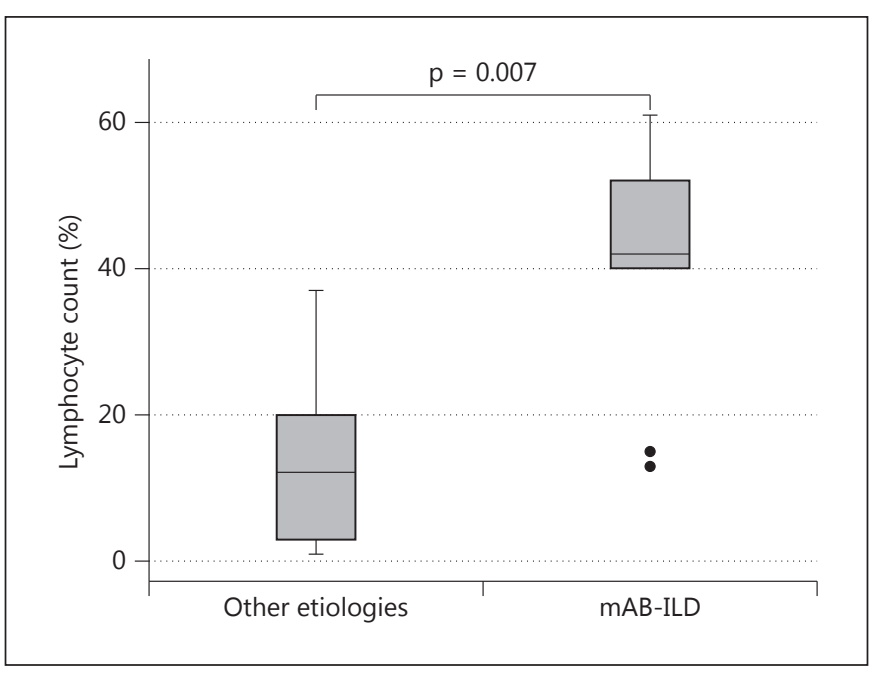

Fig. 1. Percentage of lymphocytes in BAL in mAB-ILD patients and in patients with lung disease due to other etiologies.

\section{Discussion}

To our knowledge, this is the first study describing sytematic BAL cellular analysis in patients with suspected mAB-ILD. Our study reveals that patients with $\mathrm{mAB}$-ILD present with a mixed moderate lymphocytic and mild neutrophilic alveolitis. Compared to patients on $\mathrm{mAB}$ with other lung diseases, patients with $\mathrm{mAB}$-ILD had a significantly higher percentage of lymphocytes in their BAL. We did not find any difference in CD4:CD8 ratio between the 2 groups.

In the evaluation of patients with ILD including suspected drug-induced lung disease, the utility of BAL has been well established. BAL cellular patterns are useful for narrowing the differential diagnosis in the appropriate clinical setting [5]. In patients with suspected $\mathrm{mAB}$ - 
Table 3. BAL cellular analysis

\begin{tabular}{|c|c|c|c|c|c|}
\hline & \multicolumn{2}{|c|}{ mAB-ILD $(\mathrm{n}=9)$} & \multicolumn{2}{|c|}{ Other etiologies $(n=7)$} & \multirow[t]{2}{*}{$\mathrm{p}$ value } \\
\hline & median & IQR & median & IQR & \\
\hline \multicolumn{6}{|l|}{ Cell count, \% } \\
\hline Macrophages & 38 & $30-46$ & 48 & $13-86$ & 0.5 \\
\hline Lymphocytes & 42 & $40-52$ & 10 & $3-20$ & 0.007 \\
\hline Neutrophils & 17 & $14-22$ & 10 & $1-39$ & 0.8 \\
\hline Eosinophils & 1 & $0-4$ & 1 & $0-20$ & 0.6 \\
\hline $\mathrm{CD} 4$ & 47 & $37-73$ & 25 & $9-76$ & 0.3 \\
\hline CD8 & 53 & $24-60$ & 61 & $19-92$ & 0.4 \\
\hline CD4:CD8 ratio & 0.9 & $0.6-3$ & 0.4 & $0.1-4$ & 0.3 \\
\hline Cells $/ \mathrm{ml}$ & $9.2 \times 10^{5}$ & $\begin{array}{l}9.9 \times 10^{4} \text { to } \\
1 \times 10^{7}\end{array}$ & $1.2 \times 10^{6}$ & $\begin{array}{l}1.6 \times 10^{5} \text { to } \\
6.8 \times 10^{6}\end{array}$ & 0.8 \\
\hline
\end{tabular}

ILD, previous case reports and case series have described abnormalities in the BAL fluid. Perez-Alvarez et al. [7] analyzed all case reports and case series of patients with suspected $\mathrm{mAB}$-ILD due to anti-TNF agents for autoimmune rheumatologic diseases. In 122 reports, BAL findings were available for only 12 patients. Six of the cases had lymphocytic predominance while 4 had macrophage predominance. Ramos-Casals et al. [1] published a similar study. BAL findings were reported for only 10 cases, and these showed predominantly lymphocytic alveolitis. Liote et al. [8] published a systematic review on 45 patients with rituximab-induced lung disease. BAL cellular analysis was available for 9 patients, and was consistent with lymphocytosis (13-90\%) with a predominance of CD4 lymphocytes. Arulkumaran et al. [9] described mixed neutrophilic and lymphocytic alveolitis in a case report of rituximab-induced lung disease. Sen et al. [10] reported a case of infliximab-induced interstitial pneumonitis, and they found that BAL showed CD8 lymphocyte predominance with a $\mathrm{CD} 4: \mathrm{CD} 8$ ratio of 0.74 . Most other case reports have BAL findings of either lymphocytic or mixed inflammation [11-13]. However, all of these reports described the BAL findings only in patients with suspected $\mathrm{mAB}-\mathrm{ILD}$. A direct comparison with $\mathrm{BAL}$ findings in patients on $\mathrm{mAB}$ with lung disease due to other causes was not performed.

Our study shows that patients with $\mathrm{mAB}$-ILD have an increase in the percentage of lymphocytes in the BAL fluid. The CD4:CD8 ratio in our subjects was consistent with those reported in other case series and case reports with a median CD4:CD8 ratio of 0.9 .

The presence of lymphocytic alveolitis in patients with mAB-ILD suggests the presence of a pneumonitis-type, hypersensitivity response $[4,14]$. Many drugs, including hydroxyurea, imatinib and lenalidomide, are known to cause lung disease with a hypersensitivity reaction [1518]. Consistent with this kind of reaction, all of our patients improved with discontinuation of the offending agent and administration of systemic corticosteroids. The nature of the antigenic trigger of the hypersensitivity response in these subjects is not known. Chimeric antibodies are usually made up of a murine Fab fragment which binds antigen on the target cell and a human Fc fragment which binds human immune cells for clearance of the targeted cell [19]. Either one or both components could potentially trigger the immune reaction. Detailed studies of the antigen specificity of the responding T cells are necessary to identify the triggering component of the hypersensitivity response.

Our study had certain limitations. Due to the rarity of the disease, we had a relatively small and heterogeneous group of patients. In addition, our control group consisted of patients with a variety of underlying lung diseases that could potentially affect the BAL cellular analysis. The comparison is still clinically meaningful as these diagnoses are often considered when working up patients with interstitial lung diseases. Further studies need to be conducted to establish what the 'normal' BAL cellular differential is in patients on $\mathrm{mAB}$ without any pulmonary disease.

\section{Conclusions}

Our study demonstrates that, in patients treated with $\mathrm{mAB}$, mixed inflammation with moderate lymphocytic and mild neutrophilic alveolitis is the most common BAL cellular pattern in those who develop mAB-ILD. 
The presence of lymphocytic alveolitis with a low-tonormal CD4:CD8 ratio in patients with GGOs on chest computed tomography and a good response to corticosteroids is highly suggestive that this ILD has a pneumo- nitis-type hypersensitivity reaction. In these patients, discontinuation of the $\mathrm{mAB}$, consideration of systemic corticosteroids and careful clinical follow-up can be helpful.

\section{References}

1 Ramos-Casals M, Perez-Alvarez R, Perez-deLis M, Xaubet A, Bosch X: Pulmonary disorders induced by monoclonal antibodies in patients with rheumatologic autoimmune diseases. Am J Med 2011;124:386-394.

$>2$ Favalli EG, Sinigaglia L, Varenna M, Arnoldi C: Drug-induced lupus following treatment with infliximab in rheumatoid arthritis. Lupus 2002;11:753-755.

3 Burton C, Kaczmarski R, Jan-Mohamed R: Interstitial pneumonitis related to rituximab therapy. N Engl J Med 2003;348:2690-2691.

-4 Meyer KC, Raghu G, Baughman RP, et al: An official American Thoracic Society clinical practice guideline: the clinical utility of bronchoalveolar lavage cellular analysis in interstitial lung disease. Am J Respir Crit Care Med 2012;185:1004-1014.

5 Costabel U, Uzaslan E, Guzman J: Bronchoalveolar lavage in drug-induced lung disease. Clin Chest Med 2004;25:25-35.

$\checkmark 6$ Smith PA, Kohli LM, Wood KL, Hage CA, Twigg HL 3rd, Knox KS: Cytometric analysis of BAL T cells labeled with a standardized antibody cocktail correlates with immunohistochemical staining. Cytometry B Clin Cytom 2006; $70: 170-178$.
7 Perez-Alvarez R, Perez-de-Lis M, DiazLagares $\mathrm{C}$, et al: Interstitial lung disease induced or exacerbated by TNF-targeted therapies: analysis of 122 cases. Semin Arthritis Rheum 2011;41:256-264.

8 Liote H, Liote F, Seroussi B, Mayaud C, Cadranel J: Rituximab-induced lung disease: a systematic literature review. Eur Respir J 2010;35:681-687.

-9 Arulkumaran N, Suleman R, Cecconi M, Kiely P, Chua F: Rituximab-associated pneumonitis in antineutrophil cytoplasmic antibody-associated vasculitis. J Clin Rheumatol 2012;18:39-41.

10 Sen S, Peltz C, Jordan K, Boes TJ: Infliximabinduced nonspecific interstitial pneumonia. Am J Med Sci 2012;344:75-78.

-11 Hadjinicolaou AV, Nisar MK, Parfrey H, Chilvers ER, Ostor AJ: Non-infectious pulmonary toxicity of rituximab: a systematic review. Rheumatology (Oxford) 2012;51:653662.

12 Berthod G, Lazor R, Letovanec I, et al: Pulmonary sarcoid-like granulomatosis induced by ipilimumab. J Clin Oncol 2012;30:e156-e159.
13 Achermann Y, Frauenfelder T, Obrist S, Zaugg K, Corti N, Gunthard HF: A rare but severe pulmonary side effect of cetuximab in two patients. BMJ Case Rep 2012;2012.

14 Lacasse Y, Selman M, Costabel U, et al: Clinical diagnosis of hypersensitivity pneumonitis. Am J Respir Crit Care Med 2003;168:952958.

15 Ozkan M, Dweik RA, Ahmad M: Drug-induced lung disease. Cleve Clin J Med 2001;68: $782-785$.

16 Sandhu HS, Barnes PJ, Hernandez P: Hydroxyurea-induced hypersensitivity pneumonitis: a case report and literature review. Can Respir J 2000;7:491-495.

-17 Bergeron A, Bergot E, Vilela G, et al: Hypersensitivity pneumonitis related to imatinib mesylate. J Clin Oncol 2002;20:4271-4272.

18 Thornburg A, Abonour R, Smith P, Knox K, Twigg HL 3rd: Hypersensitivity pneumonitis-like syndrome associated with the use of lenalidomide. Chest 2007;131:1572-1574.

19 Buss NA, Henderson SJ, McFarlane M, Shenton JM, de Haan L: Monoclonal antibody therapeutics: history and future. Curr Opin Pharmacol 2012;12:615-622. 